\title{
Venetoclax - The Game-changer in Hematology
}

\begin{abstract}
The introduction of small molecule inhibitors in many hematological malignancies made a landmark achievement in this field with a dramatic change in the survival outcome. Venetoclax is a B-cell lymphoma-2 inhibitor which has become the game-changer molecule in chronic lymphocytic leukemia and acute myeloid leukemia. This review is intended to summarize the mechanism of action, side effects, dosage, and different phases of clinical trials of this drug with review of literature.
\end{abstract}

Keywords: Acute myeloid leukemia, B-cell lymphoma-2 inhibitor, chronic lymphocytic leukemia, venetoclax

\section{Introduction}

Hematological malignancies such as lymphoma, acute leukemia, and multiple myeloma comprise $6.5 \%$ of all malignancies. ${ }^{[1]}$ The emergence of various targeted therapies has altered the therapeutic landscape of these diseases. Chemotherapy, the backbone of hematological malignancies, is now being gradually replaced by various targeted therapies with a better safety profile. The discovery of a new class of pro-apoptotic agents has become an important inclusion in the armamentarium to treat these cancers, irrespective of genetic diversities. ${ }^{[2]}$

Venetoclax, formerly known as ABT-199, a novel B-cell lymphoma-2 (BCL-2) inhibitor, has proven its therapeutic safety and efficacy, particularly in chronic lymphocytic leukemia (CLL) and also in acute myeloid leukemia (AML). ${ }^{[3]}$ Venetoclax is unique for its specificity for BCL-2 protein coupled with minimal hematological toxicities, making its widespread use in other low-grade BCLs. ${ }^{[4]}$ Combination therapy with this drug has also being tested in acute leukemia such as acute lymphoblastic leukemia (ALL) and aggressive lymphomas. ${ }^{[5]}$

\section{Discovery}

Professor Andrew Roberts, a hematologist from the Walter and Eliza Hall Institute

\footnotetext{
This is an open access journal, and articles are distributed under the terms of the Creative Commons Attribution-NonCommercial-ShareAlike 4.0 License, which allows others to remix, tweak, and build upon the work non-commercially, as long as appropriate credit is given and the new creations are licensed under the identical terms.
}

For reprints contact: WKHLRPMedknow_reprints@wolterskluwer.com of Australia, in 1988, found that BCL-2 protein had a role in cancer progression. ${ }^{[6]}$ His team then noticed that venetoclax had shown a remarkable response in the killing of leukemic cells in animal models. This led to the development of venetoclax as a potential cure for CLL and other hematological malignancies.

\section{Mechanism of Action}

Venetoclax is a BH-3 mimetic group of oral agents. It is a BCL-2 and BCL-XL inhibitor which promotes apoptosis. ${ }^{[7]}$ After binding with BCL-2 protein, it activates caspase with displacement of apoptotic inducers such as BIM and BAX, thereby leading to initiation of apoptotic pathway and cell killing. It has a greater affinity with BCL-2 with significantly reduced activity against BCL-XL protein which is responsible for thrombocytopenia. ${ }^{[8]}$

Approved Indications with Food and Drug Administration Approval Status

1. $17 \mathrm{p}$ deleted CLL post first-line failure - Food and Drug Administration (FDA) approval in 2016

2. CLL irrespective of the p53 status post first-line failure in 2018 gained FDA approval

3. Elderly AML in combination with hypomethylating agents - FDA approval in 2018

4. All patients with CLL in first line - FDA approval in 2019.

How to cite this article: Mandal TK, Roy S, Nayak L, Mishra BK. Venetoclax - The game-changer in hematology. Indian $\mathrm{J}$ Med Paediatr Oncol 2020;41:555-8.

\section{Tanmoy Kumar \\ Mandal, Somnath Roy, Lingaraj Nayak, B K Mishra}

Department of Medical Oncology, Tata Memorial Centre, Homi Bhabha Cancer Hospital, Varanasi, Uttar Pradesh, India

Submitted: 26-May-2020 Revised: 06-Aug-2020

Accepted: 11-Aug-2020

Published: 29-Aug-2020

Address for correspondence: Dr. Somnath Roy,

Department of Medical Oncology, Tata Memorial Centre, Homi Bhabha Cancer Hospital, Varanasi,

Uttar Pradesh, India.

E-mail: sroysskm1980@gmail. com

Access this article online Website: www.ijmpo.org DOI: 10.4103/ijmpo.ijmpo_260_20 Quick Response Code:

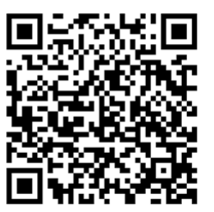




\section{Pharmacokinetics}

- Distribution: Volume of distribution - 256-321 L

- Protein binding: Highly bound to plasma protein

- Metabolism: Hepatic, predominantly via CYP3A

- Half-life (elimination): $26 \mathrm{~h}$ (approximately)

- Time to peak: 5-8 h

- Excretion: Predominantly via feces (>99.9\%).

\section{Dosage and Administration Instructions}

Tablets are available in three strengths (10 mg, $50 \mathrm{mg}$, and $100 \mathrm{mg}$ ), and it should be taken with meal and water. The dosing depends as per disease site. In CLL, an escalating dose schedule over the first 5 weeks [Table 1a] and $400 \mathrm{mg}$ once daily until unacceptable toxicity or disease progression thereafter is recommended, whereas in AML, the dose is escalated in the first 4 days along with azacitidine or decitabine or low-dose cytarabine [Table 1b]. Such kind of dose escalation strategy is adopted to debulk the tumor burden, reduce the chance of developing tumor lysis syndrome (TLS), and to check the tolerance of the drug. Venetoclax has significant drug interactions with concomitant use of CYP3A inhibitors such as imatinib, crizotinib, aprepitant, spironolactone, and various azoles (except posaconazole). Drug interactions have also been seen with P-glycoprotein inhibitors such as amiodarone, statins, and proton-pump inhibitors. The dose of venetoclax needs to be reduced by $50 \%$ when used concomitantly with these agents.

\section{Common Side Effects}

\section{Hematologic side effects}

1. Neutropenia - In patients with AML with Grade 4 neutropenia occurring upfront, the drug is not withheld. However, in patients who have post remission, then the next dose is delayed till the recovery of counts

2. Febrile neutropenia - For the first occurrence with Grade $\geq 3$ neutropenia with fever and or infection, interrupt venetoclax till the recovery of counts and then it may be restarted at the same dose level. For second and subsequent episodes, dose reduction should be done [Table 2]. Consider discontinuing venetoclax for patients who require dose reductions to $<100 \mathrm{mg}$ for more than 2 weeks

3. Anemia

4. Thrombocytopenia.

\section{Nonhematologic side effects}

1. TLS - In patients with laboratory TLS or clinical TLS post initiation of venetoclax, the drug for the next day is withheld. If the TLS requires more than $48 \mathrm{~h}$ to resolve, then the drug is restarted at a reduced dose [Table 2]. Blood biochemistry (uric acid, creatinine, potassium, phosphorus, and calcium) should be done periodically during therapy to monitor TLS parameters

\begin{tabular}{lc}
\hline $\begin{array}{c}\text { Table 1a: Escalating dose schedule in first } 5 \text { weeks in } \\
\text { chronic lymphocytic leukemia }\end{array}$ \\
\hline Week & Recommended daily dose level (mg) \\
\hline 1 & 20 \\
2 & 50 \\
3 & 100 \\
4 & 200 \\
5 & 400 \\
\hline
\end{tabular}

Table 1b: Escalating dose schedule in first 4 days in acute myeloid leukemia

\begin{tabular}{lc}
\hline Day & Recommended daily dose (mg) \\
\hline 1 & 100 \\
2 & 200 \\
3 & 400 \\
4 and beyond & 400 (with azacitidine or decitabine) \\
4 and beyond & 600 (with low-dose cytarabine) \\
\hline
\end{tabular}

Table 2: Dose de-escalation strategy for toxicity during venetoclax therapy

\begin{tabular}{lc}
\hline Dose at interruption (mg) & Restart dose (mg)* \\
\hline 400 & 300 \\
300 & 200 \\
200 & 100 \\
100 & 50 \\
50 & 20 \\
20 & 10 \\
\hline
\end{tabular}

*During the ramp-up phase, continue the reduced dose for 1 week before increasing the dose

2. Diarrhea

3. Edema

4. Upper respiratory tract infection.

\section{Landmark Trials}

\section{Phase I and II trial}

A Phase I trial was conducted to optimize the dose of venetoclax in relapsed CLL. Among the two different subgroups, the dose-escalation group was treated with dose schedules from $20 \mathrm{mg}$ weekly to $1200 \mathrm{mg}$ daily, whereas the expansion cohort group was treated with $20 \mathrm{mg}$ daily followed by a stepwise increase up to $400 \mathrm{mg}$ weekly. The overall response of the entire cohort was 79\%, with TLS reported as a dose-limiting toxicity. ${ }^{[9]}$ Its role in relapsed multiple myeloma and B-cell non-Hodgkin lymphoma (NHL) is ongoing.

A Phase II study by Jain et al. among treatment naïve high risk elderly CLL populations treated with ibrutinib monotherapy (420 mg daily) for three cycles followed by adding venetoclax (weekly dose escalation to a target dose of $400 \mathrm{mg}$ daily) from cycle four onwards. This combination therapy was given for two years' duration (total 24 cycles). At 1 year, $88 \%$ of patients were in 
complete remission and $61 \%$ achieved undetectable minimal residual disease $(\mathrm{MRD})$ in the bone marrow. ${ }^{[10]}$

\section{MURANO trial}

The Phase III randomized trial (MURANO) established the role of venetoclax with rituximab in comparison to bendamustine with rituximab with a superior overall survival and progression-free survival (PFS) benefit in patients with relapsed CLL irrespective of p53 status. ${ }^{[1]}$ The 2-year PFS rate was significantly higher (84\% vs. $36 \%$ ) in the venetoclax arm. Although the rate of Grade 3 and Grade 4 neutropenia was higher in the venetoclax and rituximab group, the rate of febrile neutropenia and infection was less in comparison with the bendamustine $\operatorname{arm} .^{[11]}$

\section{VIALE trial}

Venetoclax received accelerated approval for the treatment of elderly AML who are unfit for intensive chemotherapy based on the Phase 1 and 2 VIALE trials which compared venetoclax with low-dose azacytidine (LDAC) versus LDAC alone. ${ }^{[12]}$ This showed to have improved PFS in the venetoclax arm with superior complete remission rates.

\section{Cost-effectiveness}

The cost-effectiveness of venetoclax was measured in a cost-effective model which showed to have a lower cost than bendamustine plus rituximab- and ibrutinib-based combinations in the treatment of CLL over a 12-month duration. ${ }^{[13]}$

\section{Newer Uses in the Pipeline}

There have been recent evidences on the use of venetoclax in certain aggressive NHL such as refractory mantle cell lymphoma, ${ }^{[14]}$ relapsed diffuse large B-cell lymphoma, ${ }^{[15]}$ and relapsed follicular lymphoma. ${ }^{[16]}$ Recent clinical trials have also suggested the use of venetoclax in refractory ALL, especially T-ALL and relapsed multiple myeloma with $(11: 14)$ translocation. ${ }^{[17]}$

\section{Take-Home Points}

1. Venetoclax is the first selective BCL-2 inhibitor

2. It is approved by FDA in CLL and AML

3. Its role in other B-cell NHLs, multiple myeloma, and ALL is now being tested either in monotherapy or in combination in several clinical trials

4. It is used in escalating doses in CLL and AML.

5. TLS and neutropenia are the most common adverse reactions.

\section{Conclusion}

Venetoclax might become an important therapeutic agent against several hematological malignancies in the near future considering the acceptable toxicity profile. It is effective after failure of ibrutinib in CLL, but reverse is not true. In relapse/refractory CLL, this drug attained higher rates of MRD negativity in peripheral blood and bone marrow. We can predict that in the future, venetoclax either in monotherapy or in combination will achieve landmark milestones in the treatment of different hematological malignancies.

\section{Financial support and sponsorship}

Nil.

\section{Conflicts of interest}

There are no conflicts of interest.

\section{References}

1. Ferlay J, Colombet M, Soerjomataram I, Mathers C, Parkin DM, Piñeros $\mathrm{M}$, et al. Estimating the global cancer incidence and mortality in 2018: GLOBOCAN sources and methods. Int J Cancer 2019;144:1941-53.

2. Martens U. Small Molecules in Hematology. USA: Springer Nature; 2018.

3. Brody T. FDA's Drug Review Process and the Package Label: Strategies for Writing Successful FDA Submissions. United Kingdom: Academic Press, Elsevier Science Publishing Co.; 2017.

4. Scheffold A, Jebaraj BMC, Stilgenbauer S. Venetoclax: Targeting BCL2 in Hematological Cancers. Recent Results Cancer Res 2018;212:215-42.

5. Lenz G, Salles G. Aggressive Lymphomas. USA: Springer International Publishing; 2018.

6. Fairbrother WJ, Leverson JD, Sampath D, Souers AJ. Discovery and Development of Venetoclax, a Selective Antagonist of BCL -2. Successful Drug Discovery, edited by János Fischer et al., $1^{\text {st }}$ ed., Wiley; 2019. p. 225-45. DOI.org (Crossref),

7. Anderson MA, Deng J, Seymour JF, Tam C, Kim SY, Fein J, et al. The BCL2 selective inhibitor venetoclax induces rapid onset apoptosis of CLL cells in patients via a TP53-independent mechanism. Blood 2016;127:3215-24.

8. Ackler S, Oleksijew A, Chen J, Chyla BJ, Clarin J, Foster K, et al. Clearance of systemic hematologic tumors by venetoclax (Abt-199) and navitoclax. Pharmacol Res Perspect 2015;3:e00178.

9. Roberts AW, Davids MS, Pagel JM, Kahl BS, Puvvada SD, Gerecitano JF, et al. Targeting BCL2 with Venetoclax in Relapsed Chronic Lymphocytic Leukemia. N Engl J Med 2016;374:311-22.

10. Jain N, Keating M, Thompson P, Ferrajoli A, Burger J, Borthakur $\mathrm{G}$, et al. Ibrutinib and venetoclax for first-line treatment of CLL. N Engl J Med 2019;380:2095-103.

11. Seymour JF, Kipps TJ, Eichhorst B, Hillmen P, D'Rozario J, Assouline $\mathrm{S}$, et al. Venetoclax-rituximab in relapsed or refractory chronic lymphocytic leukemia. N Engl J Med 2018;378:1107-20.

12. A Study of Venetoclax in Combination With Azacitidine Versus Azacitidine in Treatment Naïve Subjects With Acute Myeloid Leukemia Who Are Ineligible for Standard Induction Therapy - Full Text View - ClinicalTrials.Gov. Available from: https:// clinicaltrials.gov/ct2/show/NCT02993523. [Last accessed 2020 Aug 20].

13. Davids MS, Chatterjee A, Ravelo A, Shapouri S, Manzoor BS, Sail K, et al. Cost-effectiveness of a 12-month fixed duration of venetoclax in combination with obinutuzumab in first-line chronic lymphocytic Leukemia in the United States. Blood 2019;134 Suppl 1:4741. 
14. Eyre TA, Walter HS, Iyengar S, Follows G, Cross M, Fox CP, et al. Efficacy of venetoclax monotherapy in patients with relapsed, refractory mantle cell lymphoma after Bruton tyrosine kinase inhibitor therapy. Haematologica 2019;104:e68-e71.

15. Morschhauser F, Feugier P, Flinn IW, Gasiorowski RE, Greil R, Illés Á et al. Venetoclax plus rituximab, cyclophosphamide, doxorubicin, vincristine and prednisolone (R-CHOP) improves outcomes in BCL2-positive first-line diffuse large B-cell lymphoma (DLBCL): First safety, efficacy and biomarker analyses from the phase II CAVALLI study. Blood. 2018;132(supplement 1): -782 .

16. Zinzani PL, Flinn IW, Yuen S, Topp MS, Rusconi C, Fleury I, et al. Efficacy and safety of venetoclax (Ven) + rituximab ${ }^{B}$ or Ven + Bendamustine $(\mathrm{B})+\mathrm{R}$ randomized versus $B+R$ in Patients (pts) with relapsed/refractory $(R / R)$ follicular lymphoma (FL): Final analysis of phase II CONTRALTO study. Blood 2018;132 Suppl 1:1614.

17. Wong KY, Chim CS. Venetoclax, bortezomib and S63845, an MCL1 inhibitor, in multiple myeloma. J Pharm Pharmacol 2020;72:728-37. 